\title{
Resident exposure to open simple prostatectomy in the United Kingdom: Going, going, gone? \\ (Re: National discrepancies in residency training of open simple prostatectomy for
benign prostatic enlargement: Redefining our gold standard, CUAJ, June 2020)
}

Patrick Jones, MD; Amr Hawary, MD

Department of Urology, Great Western Hospital Swindon, United Kingdom

Cite as: Can Urol Assoc J 2020;14(10):358-9. htrp://dx.doi.org/10.5489/cuaj.6794

W e read with great interest the article by LevasseurFortin et al, which provides a snapshot of exposure to open simple prostatectomy (OSP) in Canadian residency programs and valuable insight accordingly. ${ }^{1}$ In the U.K., residents are required to have carried out at least 120 transurethral resection of prostate (TURP) procedures by the end of training in order to satisfy certification requirements. However, OSP, like many other open procedures, is no longer specified.

In the U.K., a national audit commenced this year by the British Association of Urological Surgeons (BAUS), collecting data on interventions for bladder outflow obstruction (BOO). We anticipate the results for OSP will mirror those outlined by our Canadian colleagues.

Like many operations that have served as the cornerstone treatment for so many decades, OSP does so because of its efficacy. ${ }^{2}$ OSP still delivers improvements in both subjective and objective outcome measures that match robotic simple prostatectomy (RSP) or holmium laser enucleation of the prostate (HoLEP), albeit the evidence favors the latter, minimally invasive surgery in regards to blood loss and length of stay. ${ }^{3}$

However, we argue the value for resident exposure is also paramount for learning management of severe bleeding postendoscopic procedures, which in extreme circumstances, may warrant open enucleating of residual adenoma and packing of the prostate bed. Furthermore, in the case of a large benign prostate with multiple bladder stones, it can be a much quicker and simpler procedure. Indeed, many residents in the U.K. who sit the final viva exam for the Fellowship of the Royal College of Surgeons (FRCS) may have to rely on theoretical knowledge when questioned on this scenario and do not have the benefit of previous experience.

We feel that OSP remains a valuable tool in the management of benign prostatic hyperplasia in this group of patients and shouldn't be ignored in urological training.

Competing interests: The authors report no competing personal or financial interests related to this letter.

\section{References}

1. Levasseur-Fortin P, Law KW, Nguyen D-D, et al. National discrepancies in residency training of open simple prostatectomy for benign prostatic enlargement: Redefining our gold standard. Can Urol Assoc J 2020;14:182-6. http://dx.doi.org/10.5489/cuaj.6242

2. Zargooshi J. Open prostatectomy for benign prostate hyperplasia: Short-term outcome in 3000 consecutive patients. Prostate Cancer Prostatic Dis 2007;10:374-7. https://doi.org/10.1038/si.pcan.4500986

3. Elzayat EA, Ehilali MM. Holmium laser enucleation of the prostate (HoLEP): The endourologic alternative to open prostatectomy. Eur Urol 2006;49:87-91. https://doi.org/10.1016/i.eururo.2005.08.015

Correspondence: Dr. Patrick Jones, Department of Urology, Great Western Hospital Swindon, United Kingdom; patrick.jones1@nhs.net 


\title{
Author reply - Surgical management of benign prostatic obstruction: Same, same, but different
}

\author{
David-Dan Nguyen, MPH'; Kyle W. Law, BSc (Hon.)'; Kevin C. Zorn, MD, FRCSC, FACS ${ }^{2}$ \\ 'Faculty of Medicine, McGill University, Montreal, QC, Canada; '2Division of Urology, Centre hospitalier de l'Université de Montréal, Université de Montréal, Montreal, QC, Canada
}

W e thank Jones et al et al for their kind comments and interest in our work. Based on the findings from our survey sent to all Canadian urology program directors, we reported that Canadian urology residents' 2018 exposure to open simple prostatectomy (OSP) was extremely limited, likely a result of a marked decrease in volumes of OSP at academic centers, as minimally invasive approaches demonstrate their value. ${ }^{1,2}$ Similar to our findings, the authors report in their letter that there seems to be a decreasing trend in the use of OSP in the U.K., with a minimum number of OSPs that trainees must complete in order to graduate no longer specified.

Jones et al make inferences on the changing landscape of the surgical management of benign prostatic obstruction (BPO) based on publication trends. In Canada, a more direct assessment of contemporary BPO trends was recently published in CUAJ. LaBossiere et al observed that from 2002-2014, there was an increase in the use of endoscopic laser approaches with a corresponding decrease in the use of transurethral resection of the prostate (TURP), open prostatectomy, and other BPO treatments. ${ }^{3}$ Similarly, Hueber et al studied the national trends of benign prostatic hyperplasia $(\mathrm{BPH})$ management using Canadian Institute for Health Information data from 2007-2012 and identified an uptake in endoscopic laser approaches despite holmium laser and greenlight approaches remaining relatively rare compared to the U.S. ${ }^{4}$ TURP, however, remains the most common procedure for BPO. ${ }^{3,4}$ As the surgical management of BPO evolves with the introduction of novel techniques and approaches, it is only natural that medical education and resident training follow suit. A caveat to this statement is the diffusion of said novel techniques and approaches. Indeed, important considerations must be made regarding novel modalities used at academic centers and potentially taught to residents vs. what is available in the community where the majority of residents will end up practicing.
In their letter, the authors highlight the educational value of providing residents with exposure to OSP for BPO. We would also add that OSP plays an important role in the community setting, where there is more limited access to novel modalities. While we agree that some exposure to OSP is important, similarly to nearly half of Canadian urology training faculty, ${ }^{5}$ we believe that it is necessary to reassess the "Category A" designation to OSP as provided by the Royal College of Physicians and Surgeons of Canada, especially in light of the plethora of new treatment options available to men with $\mathrm{BPH}$, as approved by the CUA and other national guidelines.

Competing interests: Dr. Zorn has been a paid consultant and proctor for Boston Scientific (Greenlight, REZUM) and Procept Biorobotics (Aquablation). The remaining authors report no competing personal or financial interests related to this letter.

\section{References}

1. Levasseur-Fortin P, Law KW, Nguyen D-D, et al. National discrepancies in residency training of open simple prostatectomy for benign prostatic enlargement: Redefining our gold standard. Can Urol Assoc J 2020;14:182-6. http://dx.doi.org/10.5489/cuaj.6242

2. Nguyen D-D, Zorn KC, Bhojani N. Cost analysis of surgical options for benign disease: Implications for value. Can Urol Assoc J 2018;12:388-9. https://doi.org/10.5489/cuaj.5730

3. LaBossiere J, Wallis C, Herschorn S, et al. Surgical management of benign prostatic obstruction: 20-year population-level trends. Can Urol Assoc J 2019;14:252-7. https://doi.org/10.5489/cuaj.6224

4. Hueber PA, Zorn KC. Canadian trend in surgical management of benign prostatic hyperplasia and laser therapy from 2007-2008 to 201 1-2012. Can Urol Assoc J 2013;77:E582-6 https://doi.org/10.5489/ cuaj.203

5. Zakaria AS, Haddad R, Dragomir A, et al. Royal college surgical objectives of urologic training: A survey of faculty members from Canadian training programs. Can Urol Assoc J 2014;8:167-72. https://doi.org/10.5489/cuaj.1720

Correspondence: Dr. Kevin C. Zorn, Division of Urology, Centre hospitalier de l'Université de Montréal, Université de Montréal, Montreal, QC, Canada; zorn.chumurology@gmail.com 